\title{
Measuring UAS Pilot Responses to Common Air Traffic Clearances
}

\author{
Jason Ziccardi ${ }^{1}$, Zach Roberts ${ }^{1}$, Ryan O'Connor ${ }^{1}$, Conrad Rorie ${ }^{2}$, \\ Gregory Morales ${ }^{1}$, Vernol Battiste ${ }^{2}$, Thomas Strybel ${ }^{1}$, Dan Chiappe ${ }^{1}$, \\ Kim-Phuong L. $\mathrm{Vu}^{1}$, and Jay Shively ${ }^{2}$ \\ ${ }^{1}$ Department of Psychology, California State University Long Beach, \\ 1250 N Bellflower Blvd, Long Beach CA 90840 \\ \{jbziccardi, zach.roberts100, gregory.morales1\}@gmail.com, \\ roconnor661@hotmail.com, \\ \{thomas.strybel, dan. chiappe, kim.vu\}@csulb.edu \\ ${ }^{2}$ NASA Ames Research Center, Moffett Field, CA \\ \{conrad.rorie, vernol.battiste, robert.j.shively\} @nasa.gov
}

\begin{abstract}
Using a simulated ground control station, this study documents the methods for measuring the verbal response and execution time of unmanned aerial system (UAS) pilots to direct commands from air traffic controllers (ATCos). Although prior research has examined characteristics of ATCo-manned aircraft communication, there is very little literature on response times of UASATCo communication. Thus, there is a great need to examine the measured response of UAS pilots to ATCo commands given that there will be more extensive inclusion of UAS operations in the national airspace in the near future. The present paper aims to provide a methodology for measuring part of the UAS and ATCo interaction, one that can be used in future studies involving UAS operations in the national airspace.
\end{abstract}

Keywords: unmanned aerial systems, measured response, air traffic management.

\section{Introduction}

Unmanned aerial systems (UAS) have enormous potential for use in missions relating to scientific research, law enforcement, emergency services support, and others. In 2006, for example, NASA utilized the UAS, Ikhana, to peer through smoke and provide the U.S. Forest Service with valuable real-time forest fire imagery [1]. The technological capability of UAS have only increased since this time, and the recently passed FAA Modernization and Reform Act of 2012 [2] paves the way for more extensive integration of UAS into the national airspace.

Despite the increasing presence UAS will have, the precise manner in which they will interact with the current and future air traffic control framework has not been developed. It is assumed, though, that UAS will have to interact with air traffic control in a manner that is equivalent, in terms of safety and efficiency, to manned aircraft. This requirement will exist despite the fact that there will not be a pilot on board 
the aircraft, and that UAS have characteristics that may differ quite a bit from manned aircraft (e.g., smaller in size, slower in operating speeds, varied in terms of maneuvers that could be performed, etc.). While research into communication between manned air traffic and air traffic controllers (ATCos) exists, similar efforts have not been replicated with UAS as a focus.

Cardosi [3], for example, determined the time required for an air traffic controller (ATCo) to communicate a command to a pilot in manned aircraft in an en route environment. By reviewing voice tapes from traffic control centers and identifying traffic maneuvers, she determined the average time required for a controller to deliver a message, the average time before a pilot's response, and the duration of a pilot's acknowledgement. Cardosi found that it took, on average, approximately ten seconds for a pilot-ATCo communication to happen when accounting for the $12 \%$ of transmissions that had to be repeated due to pilot or ATCo error. Further, research has also been conducted on the effect of communication delays between manned aircraft and ATCos on controller performance and workload. Rantanen, McCarley, and Xu [4] manipulated two varieties of delay: systematic audio delay, which is the consistent delay between when the controller speaks and when it is heard in an aircraft cockpit, and pilot delay, which is the time it takes for the pilot to verbally respond following an ATCo command. They found that both audio delay and pilot delay reduced separation between aircraft in tasks where multiple communicative exchanges were required. Sollenberger, McAnulty, and Kearns [5], in a follow-up study designed to more finely determine the limits of an acceptable delay, compared a $250 \mathrm{~ms}, 350 \mathrm{~ms}$, and $750 \mathrm{~ms}$ delay, and found that a $350 \mathrm{~ms}$ delay was operationally effective for air traffic communications and resulted in the best controller performance.

Considering the lack of similar investigations involving UAS, the present study was designed to establish a methodological procedure for capturing the measured response of UAS in a simulated environment. Measured response is a measurement of UAS response times to ATCo commands, and includes the following four components: (1) time for the pilot in command of the UAS to verbally respond to ATCo instruction, (2) time for the UAS pilot to begin action after ATCo instruction, (3) time for the UAS aircraft to initiate action after the pilot's command, and (4) time for the aircraft maneuver to be visible on the controller's display. A methodology to accurately capture these intervals is integral to future experimental research investigating the communication dynamic between ATCos and UAS, and to determine critical factors that differ from that of manned aircraft. The intention is that, once established, this method could be used with more elaborate experimental manipulations to further elucidate the communications relationship that ATCOs and UAS pilots will have in the national airspace.

The present study captures the measured response by simulating the presence of UAS in an air traffic environment and recording the response time of the UAS to standard air traffic commands. Out of the four components of measured response described earlier, we focus on the measurement of the first two components: the interval between the ATCo's instruction and the UAS pilot's verbal response and the time for the UAS pilot to begin execution of ATCo command. 


\section{Method}

\subsection{Participants}

Two retired ATCos participated in the study, in addition to fourteen IFR rated pilots. The ATCos had experience in both civilian towers and civilian TRACONs. Both indicated that they were experienced with the Multi Aircraft Control System (MACS) software [6] that was used to simulate the radar, air traffic control environment. The fourteen IFR pilots did not have any UAS flying experience, but all had experience with the Multiple UAS Simulator (MUSIM) ground control station [7] used in the study. The pilots all had flight training simulator experience, and an average of 1,171 actual flight hours.

\subsection{Apparatus}

The data was collected in the Flight Deck Display Research Laboratory at NASA Ames. The MUSIM-UAS ground control station was used to simulate the actions of UAS pilots. The MUSIM display consisted of a north-up map with ownship and the current filed route of the UAS (see Fig. 1). The aircraft's path was controlled by dragging and dropping waypoints along the planned route, while altitude was controlled by editing planned altitude levels at waypoints along the planned route. During the simulation the MUSIM screen did not display any other traffic. The ATCo DSRMACS display simulated the sector used in the simulation, ZLA 20. Targets on the ATCos' DSR were depicted as a chevron icon on the display. The icon's shape and position allowed the ATCo to determine aircraft's heading and the altitude of the aircraft was displayed in its data tag. Unlike the MUSIM display, air traffic in addition to the UAS was displayed. However, the ATCo was instructed to only pay attention to the traffic when instructed to issue a traffic call to the UAS. In other words, the ATCos were not managing the traffic in the sector. The pilot, ATCo, and experimenters that were facilitating the data collection spoke to each other using push-to-talk headsets over a voice system.

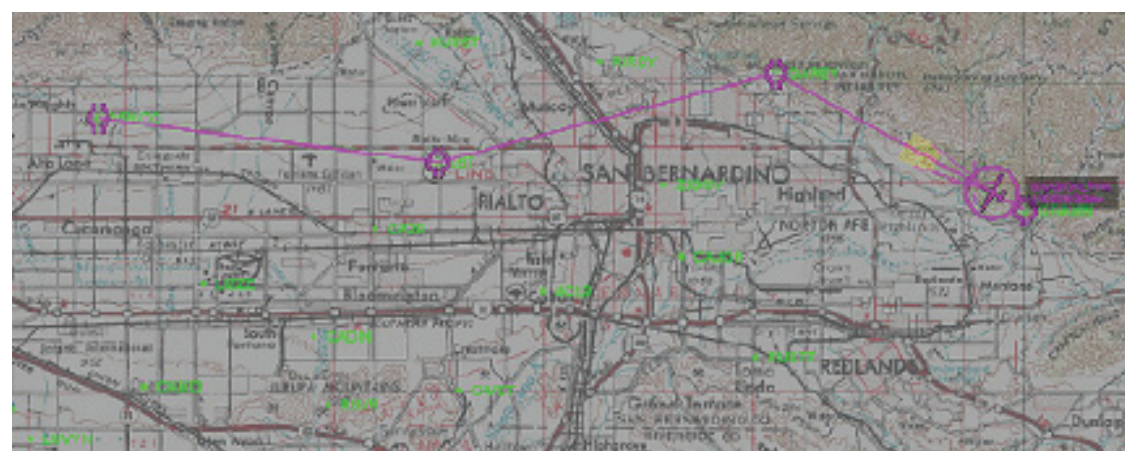

Fig. 1. MUSIM-UAS map display with a waypoint path for the UAS specified 


\subsection{Procedure}

Pilots participated in this data collection effort following an unrelated study that used MUSIM, so virtually no training in MUSIM operation was needed. Regardless, pilots received a five minute training presentation to familiarize them with the simulation and reviewed important MUSIM procedures. Following the training presentation and prior to the experimental trials, each pilot completed an approximately five minute training scenario during which they received five different commands that included two frequency changes, one altitude change, one heading change, and one command that involved editing a waypoint. These commands were pulled from a list of 15 base commands, which were presented in a partially counterbalanced order. The commands consisted of crossing restrictions, traffic calls, radio frequency changes, route amendments and direct to waypoint clearances. The following are examples of each the 15 command types used, presented here in an arbitrary order:

- "PD-1, cross GAREY at one-four-thousand."

- "PD-1 turn left heading two-four-zero, proceed direct ZIGGY, then resume own navigation."

- "PD-1, descend and maintain one-two-thousand."

- "PD-1, turn right, fly heading one-two-zero."

- "PD-1 climb and maintain one-five-thousand, traffic twelve o'clock, five-zero miles, westbound one-three-thousand."

- "PD-1, contact center 133.25."

- "PD-1, traffic nine o'clock, five-zero miles, eastbound one-four-thousand. Turn right immediately, fly heading one-five-zero."

- "PD-1, contact tower 129.00."

- "PD-1, traffic one o'clock, five-zero miles, westbound one-five-thousand. Turn left immediately, fly heading two-six-zero."

- "PD-1, contact approach 126.30."

- "PD-1, descend and maintain one-three-thousand. Traffic eleven o'clock, five-zero miles, eastbound one-four-thousand."

- "PD-1, turn left, fly heading one-eight-zero."

- "PD-1, climb and maintain one-six-thousand."

- "PD-1, turn right three-one-zero, direct KRAIL, then resume own navigation."

- "PD-1, cross CAJON at one-five-thousand."

As noted earlier, the commands were partially counterbalanced between participants, and each participant completed two identical trials of fifteen commands. The ATCo was provided with a list of commands for each trial. However, in their list, some commands were missing specific information (which had to be changed on a trial-totrial basis depending on the current UAS location), such as the waypoint to direct the UAS. The missing information was provided during the trial by an experimenter seated adjacent to the ATCo. Each command was issued after the MUSIM observer confirmed the MUSIM pilot was ready. The ATCo would issue each command individually and the pilot would verbally acknowledge the command and begin its execution. As the aircraft started the action on the ATCo scope, the ATCo would confirm 
the command was being executed on his MACS-DSR screen, and this signaled the end of the trial. The MUSIM pilot was instructed to focus only on completing ATCo commands as quickly and accurately as possible, and s/he was not performing any other UAS relevant task.

\section{Data Collection Method}

Timestamps were collected for verbal exchanges between ATCo and UAS pilot communication, UAS pilot actions on the MUSIM station, and observable indications of aircraft changes on video recordings of both the pilot's MUSIM display and ATCo's MACS radar displays. For each of the fifteen commands, these data allowed a detailed and accurate timeline of ATCo and UAS verbalizations and actions. In addition to the timing data, pilot workload and ATCo acceptability ratings were also collected, but this data is not being reported here.

\subsection{Verbal Exchange Timing Data}

Verbal exchange data that were collected included the time when the ATCos and pilots depressed and released the push-to-talk button on their headset, as well as the time that they actually started and stopped speaking. The timing data for the push-totalk button were collected from a voice logger program that provided exact time in UTC of button presses that was written to a text output file (see Fig. 2). These UTC times were synched with a voice recording of the trial and coded as different aspects of the exchange between pilot and ATCo (ATCo beginning verbal clearance, ATCo ending verbal clearance, UAS pilot beginning verbal response, etc). Timing data for actual speech were determined by listening to a video file that displayed UTC synchronous with an audio recording of the exchanges.

Using this method, the following time points were collected for each command:

- Time ATCo depressed/released push-to-talk button in order to deliver clearance, and the time the ATCo verbally began speaking and when the ATCo ended issuing the clearance.

- Time pilot depressed/released push-to-talk button for acknowledgement of the ATCo command was collected in addition to the time the pilot verbally began speaking and the pilot's verbal response ended.

- Time that the ATCo depressed/released push-to-talk button, which signaled the detection of the executed command on the ATCo screen.

In the event that the command was a frequency change, timing data related to the pilot checking out of the original frequency and checking in to the new frequency, in addition to the response of the ATCo on the new frequency, were collected. All of these data were used to determine the interval for the first component of measured response: the time for the pilot in command of the UAS to verbally respond to ATCo instruction. 


\begin{tabular}{|l|r|r|r|l|l|}
\hline 4 & \multicolumn{1}{|c|}{ A } & B & \multicolumn{1}{c|}{ C } & \multicolumn{1}{|c|}{ D } & \multicolumn{1}{|c|}{ E } \\
\hline 1 & JulianTime(Sec) & MonthDay & UTCtime VoicelD & ON/OFF \\
\hline 2 & 1344027493 & 3-Aug & $20: 58: 13$ & SimMgr & ON \\
\hline 3 & 1344027499 & 3-Aug & $20: 58: 19$ SimMgr & OFF \\
\hline 4 & 1344027501 & 3-Aug & $20: 58: 21$ & Ghost18 & ON \\
\hline 5 & 1344027503 & 3-Aug & $20: 58: 23$ & SimMgr & ON \\
\hline 6 & 1344027503 & 3-Aug & $20: 58: 23$ & Ghost18 & OFF \\
\hline 7 & 1344027507 & 3-Aug & $20: 58: 27$ SimMgr & OFF \\
\hline 8 & 1344027511 & 3-Aug & $20: 58: 31$ kaitlin laptop & ON \\
\hline 9 & 1344027513 & 3-Aug & $20: 58: 33$ & SimMgr & ON \\
\hline 10 & 1344027513 & 3-Aug & $20: 58: 33$ kaitlin laptop & OFF \\
\hline 11 & 1344027515 & 3-Aug & $20: 58: 35$ SimMgr & OFF \\
\hline 12 & 1344027517 & 3-Aug & $20: 58: 37$ UasPilot & ON \\
\hline
\end{tabular}

Fig. 2. Output from logger program that recorded exact UTC time of button presses used to initiate and end voice transmission

\subsection{MUSIM Pilot Actions Timing Data}

The actions that the pilots executed on the MUSIM station in response to the ATCo commands were coded, time stamped and recorded in a text output file. Using this output (see Fig.3), the exact time the pilots began and ended executions were recorded. For frequency changes, a video recording that displayed UTC time in addition to the frequency change panel was used to visually determine timing. These data were used to determine the interval for the second component of measured response: the time for the UAS pilot to begin an action after completion of the ATCo's instruction.

\begin{tabular}{|c|l|r|l|l|}
\hline 5 & Type & & GMT Time & Event \\
\hline 6 & $-\ldots-$ & & $-1 .--$ &.--- \\
\hline 7 & DCEvent: & $21: 00: 15$ & 22.133302 & Path initially selected. \\
\hline 8 & DCEvent: & $21: 00: 19$ & 27.066642 & Path commit received: 1. \\
\hline 9 & DCEvent: & $21: 00: 48$ & 56.033299 & Path initially selected. \\
\hline 10 & DCEvent: & $21: 00: 52$ & 59.766654 & Path commit received: 2. \\
\hline 11 & DCEvent: & $21: 01: 26$ & 93.53332 & Path initially selected. \\
\hline 12 & DCEvent: & $21: 01: 30$ & 97.433299 & Path commit received: 3. \\
\hline 13 & DCEvent: & $21: 02: 40$ & 167.233298 & Path initially selected. \\
\hline 14 & DCEvent: & $21: 02: 44$ & 171.166639 & Path commit received: 4. \\
\hline 15 & DCEvent: & $21: 03: 37$ & 225.033319 & Path initially selected. \\
\hline
\end{tabular}

Fig. 3. Output from MUSIM station detailing pilot actions and execution times

\section{Discussion}

By Using the MUSIM-UAS and MACS-DSR simulation software in conjunction with the method described above, we collected timing data for various aspects of UAS pilot responses to an (FAA suggested) limited number of air traffic controller commands/clearances. With regards to the timing data, two components of the pilots' 
response to ATCo commands were designed to be accurately captured for further analysis: the time for the pilot in command of the UAS to verbally respond to ATCO instruction, and the time for the UAS pilot to begin action after ATCo instruction. The success of this method of data extraction provides a methodology for further experimental studies investigating ATCo and UAS pilot communication.

Acknowledgments. This project was supported by in part by NASA cooperative agreement NNX12AH23A, NASA UAS in the NAS (Walter Johnson, Technical Monitor), and NASA cooperative agreement NNX09AU66A, Group 5 University Research Center: Center for Human Factors in Advanced Aeronautics Technologies (Brenda Collins, Technical Monitor).

\section{References}

1. Ambrosia, V.G., Wegener, S., Zajkowski, T., Sullivan, D.V., Buechel, S., Enomoto, F., Lobitz, B., et al.: The Ikhana unmanned airborne system (UAS) western states fire imaging missions: from concept to reality (2006-2010). Geocarto International 26(2), 85-101 (2010)

2. $112^{\text {th }}$ Congress, FAA Modernization and Reform Act of 2012 (2012), http : / / www . gpo.gov/fdsys /pkg/CRPT-112hrpt381/pdf / CRPT-112hrpt381.pdf (retrieved)

3. Cardosi, K.M.: Time Required for Transmission of Time-Critical Air Traffic Control Messages in an En Route Environment. The International Journal of Aviation Psychology 3(4), 303-314 (1993)

4. Rantanen, E.M., McCarley, J.S., Xu, X.: Time Delays in Air Traffic Control Communication Loop: Effect on Controller Performance and Workload. The International Journal of Aviation Psychology 14(4), 369-394 (2004)

5. Sollenberger, R.L., McAnulty, M., Kerns, K.: The Effect of Voice Communications Latency in High Density. Communications-Intensive Airspace (2003)

6. Prevot, T.: Exploring the many perspectives of distributed air traffic management: The Multi Aircraft Control System: MACS. In: International Conference on Human-Computer Interaction in Aeronautics, HCI-Aero 2002, pp. 23-25 (2002)

7. Fern, L., Shively, J.: Designing airspace displays to support rapid immersion for UAS handoffs. In: Proceedings of the Human Factors and Ergonomics Society 55th Annual Meeting, pp. 81-85. Human Factors and Ergonomics Society, Santa Monica (2011) 